\title{
Isolation and Characterization of Microcrystalline Cellulose (MCC) from Rice Husk (RH)
}

\author{
Zuliahani Ahmad ${ }^{1, a}$, Nurul Nadhirah Roziaizan ${ }^{1}$, Rozyanty Rahman ${ }^{2}$, Ahmad Faiza Mohamad ${ }^{3}$ and \\ Wan Izhan Nawawi Wan Ismail ${ }^{1}$ \\ ${ }^{1}$ Faculty of Applied Sciences, Universiti Teknologi Mara, 02600 Arau, Perlis, Malaysia \\ ${ }^{2}$ School of Material Engineering, Universiti Malaysia Perlis, 02600 Arau, Perlis, Malaysia \\ ${ }^{3}$ Faculty of Industrial Technology, Universiti Teknologi Mara, 40450 Shah Alam, Selangor, Malaysia
}

\begin{abstract}
Microcrystalline cellulose (MCC) was extracted from local agricultural residues, rice husk (RH). RH undergone alkaline, bleaching and acid hydrolysis treatments. Nitric acid was used in the hydrolysis process as a potential acid to replace strong acids such as hydrochloric and sulphuric acids. Hydrolysis of RH was carried out by using different molarity of nitric and hydrochloric acids (control). The properties of MCC obtained such as \% yield, crystallinity index $(\mathrm{CrI})$ and functional group present were studied. \% yield shown a comparable result regardless of different acid used. Whilst Fourier transform infrared (FTIR) spectroscopy showed the progressive removal of non-cellulosic constituents. X-ray diffraction (XRD) analysis revealed that the crystallinity increased with successive treatments regardless different molarity and acids used. The results showed potential of nitric acid to be used in MCC isolation process.
\end{abstract}

\section{Introduction}

A lot of rice husk $(\mathrm{RH})$ is generated during the milling process in rice milling industries. $78 \%$ of the paddy is received as bran, broken rice and rice. Other $22 \%$ of the paddy received as husk. Generally, $\mathrm{RH}$ made up of 33\% cellulose, $26 \%$ hemicelluloses and 7\% lignin [1]. Most of the RH widely used in building material, fertilizer, insulation material, oil spill absorbent, heat insulators as well as a fillers in composite preparation [2,3]. Nevertheless, large quantities of $\mathrm{RH}$ are usually burn in an open air, which creates environmental issues. Therefore, it is necessary to reduce environmental problem by fully utilize RH as cellulose derivatives source.

Cellulose is one of the biodegradable and renewable structural plant polymer which can be processed into whisker-like micro fibrils, microcrystalline cellulose (MCC), nanocrystalline cellulose (NCC) and many more [4,5]. It can be obtained from agricultural by-products such as cotton fiber, kenaf, hemp, corn stalk, bagasse and RH. MCC exhibits excellent properties in composite fabrication such as renewability, biodegradability, high surface area for bonding with resins [4-6].

The isolation technique is called hydrolysis method where the amorphous region of cellulosic material is disintegrated resulting with different degrees of crystallinity index. Many researchers reported on using strong minerals acids such as sulphuric acid ( $\mathrm{H} 2 \mathrm{SO} 4)$ and hydrochloric acid $(\mathrm{HCl})$ hydrolyzed the amorphous regions [6-7]. In this study, HNO3 has been chosen as an alternative to the

${ }^{\text {a }}$ Corresponding author : zuliahani@perlis.uitm.edu.my 
strong acid because it is completely miscible with water, only small amounts needed to catalyze the hydrolysis reaction and less corrosive. The significance of this research is to encourage the utilization of MCC isolated from $\mathrm{RH}$ in composite preparation via acid hydrolysis using $\mathrm{HNO} 3$ in comparison with $\mathrm{HCl}$.

\section{Materials}

The rice husk was collected from BERNAS, a local rice mills in Simpang Empat, Perlis. $\mathrm{NaOCl}$ and $\mathrm{NaOH}$ were used as bleaching agents while $\mathrm{HNO} 3$ and $\mathrm{HCl}$ were used for hydrolysis. All the chemicals was purchased from Fluka and was used without further purification.

\subsection{Pre-treatment of RH}

The alkaline treatment was performed to solubilise the hemicelluloses. $10 \mathrm{~g}$ of $\mathrm{RH}$ were refluxed with $120 \mathrm{~mL}$ of $1 \mathrm{M} \mathrm{NaOH}$ at $80^{\circ} \mathrm{C}$ for $1 \mathrm{~h}$ and $36 \mathrm{~min}$. Following alkali treatment, the alkaline treated $\mathrm{RH}$ were refluxed with $140 \mathrm{~mL}$ of $5 \%$ sodium hypochlorite $(\mathrm{NaOCl})$ at $80{ }^{\circ} \mathrm{C}$ for $18 \mathrm{~min}$ [8] in the bleaching process. The lignin become soluble in the alkaline medium and oxidized during bleaching.

\subsection{Acid hydrolysis process}

$10 \mathrm{~g}$ of pre-treatment $\mathrm{RH}$ were treated with $61 \mathrm{~mL}$ of $1 \mathrm{M}$ and $2 \mathrm{M} \mathrm{HNO} 3$ at room temperature for 30 min under continuous stirring. It was washed repeatedly with distilled water until $\mathrm{pH} 7$ is achieved. The resultant MCC were grounded into a fine powder by using rotary ball mill after the washing and drying process. The mass of dried RH were recorded and the same steps were used for $1 \mathrm{M}$ and $2 \mathrm{M}$ $\mathrm{HCl}$.

\subsection{Percentage yield}

Percentage yield was calculated using the following equation:

$$
\text { Percent yield }(\%)=\frac{\text { weight initial }}{\text { weight after hydrolysis }} \times 100 \%
$$

\subsection{Fourier transform infrared (FTIR) spectroscopy}

FTIR analysis was carried out using Perkin Elmer FTIR spectrometer 1650 with scanning ranges of $4000.0 \mathrm{~cm}^{-1}$ to $400.0 \mathrm{~cm}^{-1}$ at room temperature.

\subsection{X-ray diffraction (XRD)}

X-ray diffraction was performed to determine crystallinity index at different treatments. Diffraction patterns were obtained using a PANalytical X'PertPRO Multi-Purpose Diffractometer with $\mathrm{Cu} \mathrm{K} \alpha$ radiation. The crystallinity index (CrI) were calculated via Equation (2) [9]:

$$
\mathrm{CrI}=\left(\frac{I_{002}-I_{a m}}{I_{002}}\right)
$$

where $\mathrm{I}_{002}=$ intensity of the 002 peak (at about $2 \theta=26$ ) $\mathrm{I}_{\mathrm{am}}=$ intensity corresponds to the peak at about $2 \theta=8$. 


\section{Results and Discussion}

\subsection{Percentage yield}

The yield of MCC sample was determined and the data was summarized in Table 1. Different molarity and acids were used to extract $\mathrm{MCC}$ from $\mathrm{RH}$ which were $1 \mathrm{M} \mathrm{HNO}_{3}, 1 \mathrm{M} \mathrm{HCl}, 2 \mathrm{M} \mathrm{HNO}$ and $2 \mathrm{M} \mathrm{HCl} .1 \mathrm{M}$ and $2 \mathrm{M} \mathrm{HCl}$ gave $60.58 \%$ and $69.24 \%$ while $1 \mathrm{M}$ and $2 \mathrm{M} \mathrm{HNO}_{3}$ gave $80.64 \%$ and $83.26 \%$ of MCC yield respectively. Higher molarity of acids gave higher MCC yield. This is due to to at higher acid concentration, more solubilised amorphous region of cellulose contribute to increase $\%$ of MCC yield. The percentage yield of MCC varied from sample to sample. Also, the difference was depending on the type of paddy, climatic, geographical conditions, sample preparation and method of analysis which could be the reason for this variation [7]. As a conclusion, $1 \mathrm{M} \mathrm{HNO}_{3}$ and $2 \mathrm{M} \mathrm{HNO}_{3}$ gave higher $\mathrm{MCC}$ yield as compared to $\mathrm{HCl}$ regardless of molarity.

Table 1. Yield of MCC sample at different molarity and acids used.

\begin{tabular}{|c|c|}
\hline Samples & \% Yield \\
\hline $1 \mathrm{M} \mathrm{HNO}_{3}$ & 80.64 \\
\hline $1 \mathrm{M} \mathrm{HCl}$ & 60.58 \\
\hline $2 \mathrm{M} \mathrm{HNO}_{3}$ & 83.26 \\
\hline $2 \mathrm{M} \mathrm{HCl}$ & 69.24 \\
\hline
\end{tabular}

\subsection{FTIR}

Figure 1 shows the FTIR spectra recorded for untreated RH and RH treated with different acids. All samples display two main absorbance regions at $2900-3500 \mathrm{~cm}^{-1}$ and $450-1750 \mathrm{~cm}^{-1}$ in line with Rosa et. al. [10] corresponding to $\mathrm{OH}$ bending, $\mathrm{CH}_{2}$ groups, $\mathrm{C}-\mathrm{O}-\mathrm{C}$ stretching and $\mathrm{C}-\mathrm{H}$. The peak at 3350$3440 \mathrm{~cm}^{-1}$, which was observed in all spectras, represents of the $\mathrm{C}-\mathrm{H}$ and $\mathrm{O}-\mathrm{H}$ groups. The absorption at region from 1637 until $1651 \mathrm{~cm}^{-1}$ in all samples are corresponding to the absorption of water. According to Nurain Johar et. al. [7], this band is related to bending modes of water molecules due to a strong interaction between cellulose and water. This indicates that the cellulose components were present after the chemical treatment carried out on the rice husk fibres.

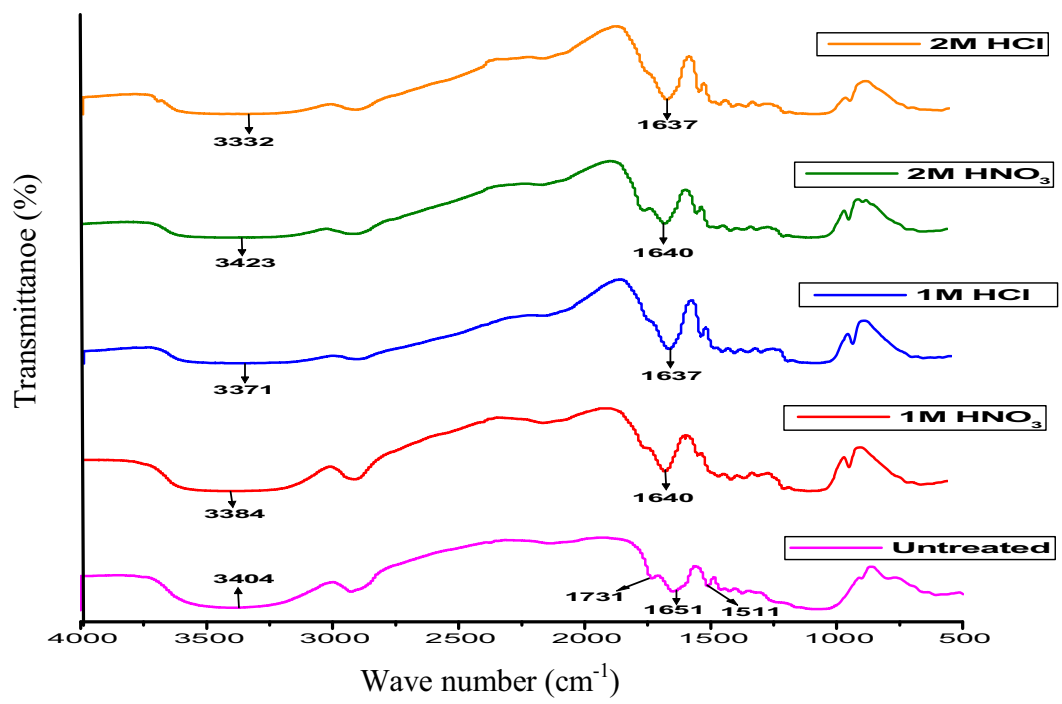

Figure 1. FTIR spectra of untreated rice husk and treated rice husk. 


\section{MATEC Web of Conferences}

In addition, Rosa et. al. [10] found the absence of peaks located in the range of 1509-1609 $\mathrm{cm}^{-1}$, which correspond to the $\mathrm{C}=\mathrm{C}$ aromatic skeletal vibrations indicate the complete removal of lignin. The absorption band which corresponds to the acetyl or ester groups of hemicelluloses appears in the region $1700-1740 \mathrm{~cm}^{-1}$ as can be seen in untreated $\mathrm{RH}$. Whilst the absence of this peak was found in treated RH indicates the removal of hemicelluloses was successfully done [10-11]. The peak located at $1049-1079 \mathrm{~cm}^{-1}$ was observed in five spectras referring to the $\mathrm{C}-\mathrm{H}$ stretching vibration of $\mathrm{C}-\mathrm{O}$ [12]. Based on FTIR spectras, lignin and hemicelluloses have been removed via bleaching treatment, alkali treatment and acid hydrolysis. In addition, no significance difference were observed in all samples at the cellulose region, which indicates the cellulose molecular structure remains unchanged from the treatment.

\subsection{XRD}

Figure 2 shows the diffraction patterns obtained for untreated and treated rice husk with different molarities and acid used. The peak at $2 \theta=16^{\circ}, 22.5^{\circ}$ and $36^{\circ}$ is correspond to cellulose I region for all samples. This is supported by Rosa et al. [10] and Satyamurthy et al. [13] which there are typical of cellulose I with three well-defined crystalline peaks around $2 \theta=16^{\circ}, 22.5^{\circ}$ and $36^{\circ}$ and indicated by the absence of the doublet located at $22.5^{\circ}$.

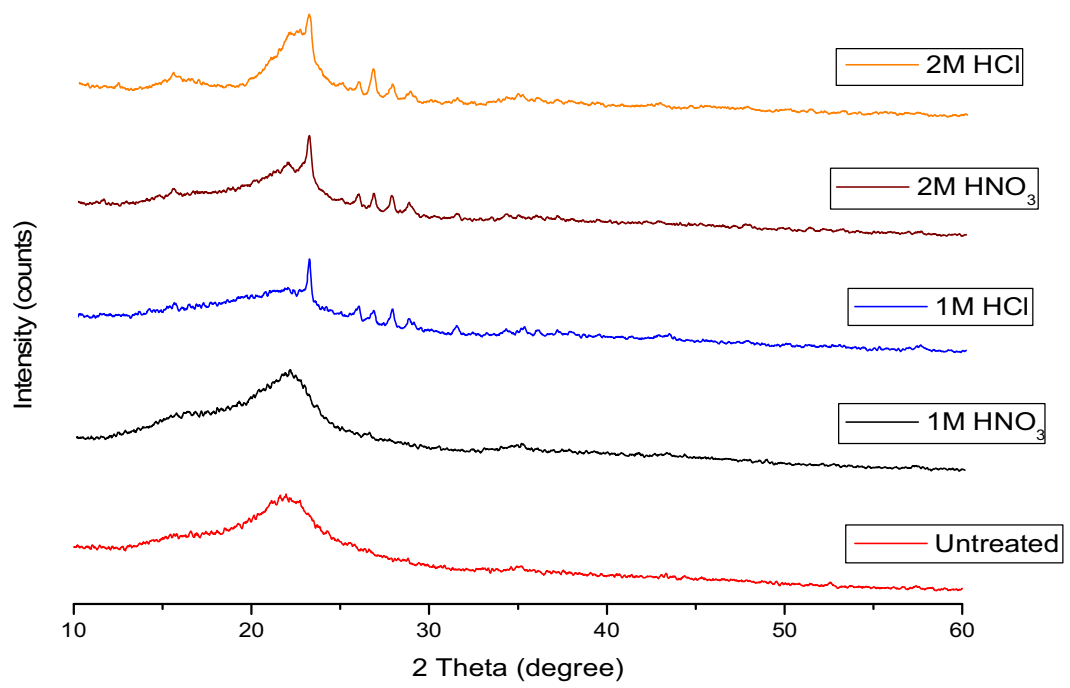

Figure 2. X-ray diffraction patterns of untreated and treated rice husk with different molarities.

The crystallinity index was determined for various samples and the results are summarized in Table 2 . The crystallinity values of treated $\mathrm{RH}$ with $2 \mathrm{M} \mathrm{HNO}_{3}$ is $63.9 \%$ which is higher compared to others. It is due to the removal of amorphous regions of cellulose by acid hydrolysis, which prompts the hydrolytic cleavage of glycosidic bonds, finally releasing individual crystallites [6]. In comparison, $2 \mathrm{M} \mathrm{HNO}_{3}$ and $2 \mathrm{M} \mathrm{HCl}$ shows comparable result and $1 \mathrm{M} \mathrm{HNO}_{3}$ gives higher percentage crystallinity compared to $1 \mathrm{M} \mathrm{HCl}$.

During the hydrolysis process, hydronium ions can penetrate the more accessible amorphous regions of cellulose and allow the hydrolytic cleavage of glycosidic bonds, which eventually releases individual crystallites [7]. In addition, during the preparation of cellulose the growth and realignment of monocrystals may occur in parallel and thus can improve the cellulose crystallinity [7]. An increase in crystallinity is related to increases in the rigidity of the cellulose structure, which can lead to higher tensile strength to fibers. This increase would be expected to enhance the mechanical properties of composites [10]. 
Table 2. Crystallinity index of treated and untreated RH.

\begin{tabular}{|c|c|}
\hline Sample & Crystallinity (\%) \\
\hline Untreated $\mathrm{RH}$ & 39.3 \\
\hline $\mathrm{MCC}-1 \mathrm{M} \mathrm{HNO}_{3}$ & 42.0 \\
\hline $\mathrm{MCC}-1 \mathrm{M} \mathrm{HCl}^{(\%)} \mathrm{HNO}_{3}$ & 37.0 \\
\hline $\mathrm{MCC}-2 \mathrm{M} \mathrm{H} \mathrm{HCl}^{2}$ & 63.9 \\
\hline $\mathrm{MCC}-2 \mathrm{M} \mathrm{HCl}$ & 52.2 \\
\hline
\end{tabular}

\section{Conclusion}

MCC has been successfully isolated from RH using chemical treatments involving alkali, bleaching and acid hydrolysis treatments. The results obtained from FTIR analysis confirmed that chemical structure of cellulosic fragments is not influenced by the acid hydrolysis. The crystallinity analysis shows that MCC give high crystallinity providing evidence that acid hydrolysis does not alter crystal structure from cellulose I of $\mathrm{RH}$. The proposed $\mathrm{HNO}_{3}$ gives comparable \% yield and \% crystallinity as compared to $\mathrm{HCl}$ making it a potential acid to be used in acid hydrolysis process.

\section{References}

[1] A.M. Adel, H.Z. Abd El-Wahab, A.A. Ibrahim and M.T. Al-Shemy, Characterization of microcrystalline cellulose prepared from lignocellulosic materials, Part II: Physicochemical properties. Carbohydrate Polymers, 83, 676-687, (2011).

[2] O. Olamide and F.A. Oyawale, Characterization of rice husk via atomic absorption spectrophotometer for optimal silica production, Int. J. of Science and Technology, 2(4), 210-213, (2012).

[3] C.P. Azubuike and A.O. Okhamafe, Physicochemical, spectroscopic and thermal properties of microcrystalline cellulose derived from corn cobs, Int. J. of Recycling of Organic Waste in Agriculture,1(9), 1-7, (2012).

[4] E. Abraham, B. Deepa, L.A. Pothen, J. Cintil, S. Thomas, M.J. John, R. Anandjiwala and S.S. Narine, Enviromental friendly method for the extraction of coir fibre and isolation of nanofibre, Carbohydrate Polymers, 92, 1477-1483, (2013).

[5] S.J. Eichhorn, Cellulose nanowhiskers: Promising materials for advanced applications, Soft Matter, 7, 303-315, (2011).

[6] M.K. Mohamad Haafiz, S.J. Eichhorn, A. Hassan and M. Jawaid, Isolation and characterization of microcrystalline cellulose from oil palm biomass residue, Carbohydrate Polymers, 93, 628-634, (2013).

[7] N. Johar, A. Dufresne and I. Ahmad, Extraction, preparation and characterization of cellulose fibers and nanocrystals from rice husk, Industrial Crops and Products, 37, 93-99, (2012).

[8] A.P. Mario, D.W. Marseno and Haryadi, Synthesis and characterization of sodium carboxymethylcellulose from cavendish banana pseudo stem (Musam cavendishii Lambert), Carbohydrate Polymers, 62, 164-169, (2005).

[9] A.M. Adel, H.Z. Abd El-Wahab, A.A. Ibrahim and M.T. Al-Shemy, Characterization of microcrystalline cellulose prepared from lignocellulosic materials, Part I: Acid Catalyzed Hydrolysis, Biosource Technology, 101, 4446-4455, (2010). 


\section{MATEC Web of Conferences}

[10] S.M.L. Rosa, N. Rehman, M.I.G. De Miranda, S.M.B. Nachtigall and C.I.D. Bica, Chlorine-free extraction of cellulose from rice husk and whisker isolation, Carbohydrate Polymers, 87, 1131$1138,(2012)$.

[11] M. Nuruddin, A. Chowdhury, S.A. Haque, M.,Rahman, S. F. Farhad, M. Sarwar Jahan and A. Quaiyyum, Extraction and characterization of cellulose nanofibrils from agricultural wastes in an integrated biorefinery initiative, Cellulose Chemical and Technology, 45, 347-354, (2011).

[12] A. Alemdar and M. Sain, Biocomposites from wheat straw nanofibres: Morphology, thermal and mechanical properties, Composites Science and Technology, 68, 557-565, (2008).

[13] P. Satyamurthy, P. Jain, R.H. Balasubramaya and N. Vigneshwaran, Preparation and characterization of cellulose nanowhiskers from cotton fibres by controlled microbial hydrolysis, Carbohydrate Polymers, 83, 122-129, (2011). 